\title{
Study on the process of droplet formation when liquid flows out of a capillary
}

\author{
Muksin Khodjiev ${ }^{1 *}$, and Orif Alimov ${ }^{2}$ \\ ${ }^{1}$ Gulistan State University, Gulistan, Uzbekistan \\ ${ }^{2}$ Jizzakh Polytechnic Institute, Jizzakh, Uzbekistan
}

\begin{abstract}
In this article, the law of interaction of air and fibrous materials emanating from two opposite pipelines is described in the theory of singular points by S.A. Chapligin, N.E. Zhukovsky function, theoretically studied using K. Schwarz's integral formula, Lopital's rule, complex potential field and canonical field.
\end{abstract}

\section{Introduction}

It is known that the air flow is widely used in the technological process of cotton processing. The air flow is widely used [1], especially when transporting cotton, fiber, and also when transporting waste from technological processes. In this area, B. Levkovich, O. Ishmurotov, S. Kadyrkhodzhaev, A. Suslin, R. Burnashev, M. T. Khodzhiev, R. Murodov, B. Mardonov and Kh. Akhmedkhodzhaev and others conducted extensive scientific and practical research on the transportation of cotton products in pneumatic vehicles, but studies of pneumatic vehicles used for dust removal and studies of dust concentrations emitted from dust collectors have not been sufficiently conducted [2-13].

With this in mind, today, in the in-depth study of the composition of dust, great attention should be paid to the issue of separating their constituents during the cleaning process [1]. In particular, the analysis of the existing technology for dust cleaning of air shows that no scientific and practical studies of the cleaning process, taking into account the fractional composition of dusty air, have not been carried out.

The problem of air flow and separation of fibrous materials from all of the above technological processes is a very urgent problem, and its solution is extremely important.

In solving this problem, we tried to create a technology for their separation, based on a sharp decrease in the speed of heavy particles in the air, based on the force of interaction of oppositely directed air flows [2].

The creation of a theoretical study of this process is one of the important tasks. To do this, we will take into account the regularity of the interaction of air and a fibrous mixture coming from two opposite tubes in this process. In it, we consider a theoretical study of this issue, mainly based on the technological scheme presented in Fig. 1 [3].

* Corresponding author: glsu_rektor@edu.uz 


\section{Materials and Methods}

A mixture of two pipes in a horizontal position collides with each other during movement and propagates along the upper and lower vertical pipes, as shown in the diagram in Figure 1. As a result of the collision of opposite currents around the point of their collision E, a steady state current is formed (Fig. 2 and 3). As a result, the state of suspension formation in Fig. $2\left(C_{0} E D_{0}\right)$, the symmetric state of the two mean currents of the $\mathrm{G}_{\mathrm{z}}$ was investigated on the basis of the circuit shown in Fig. 3.

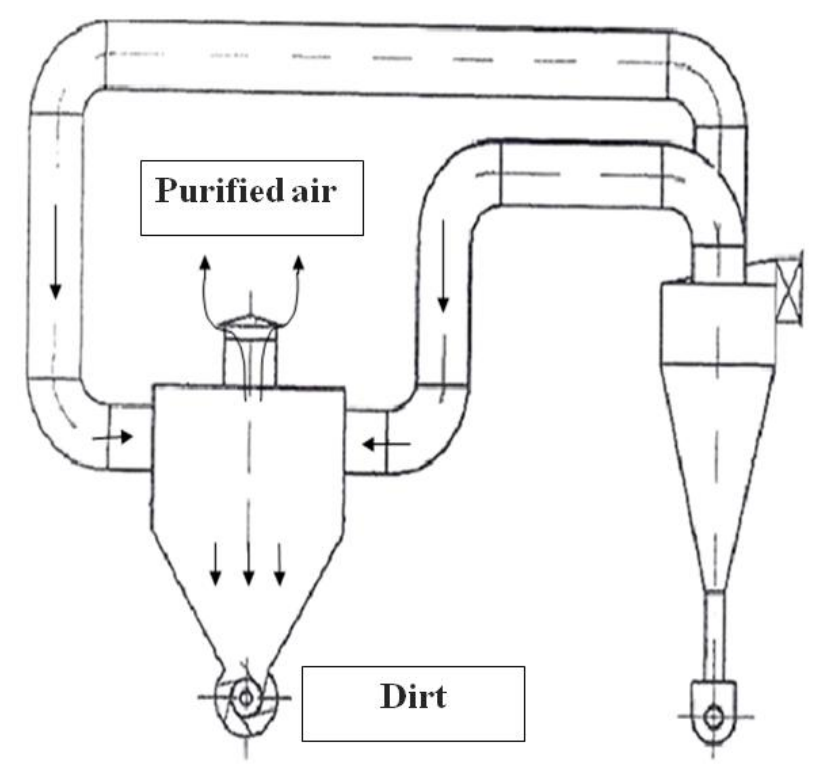

Fig.1. Diagram of the interaction of air and fiber mixture coming from two opposite pipes

As a result of the study, the following indicators and their parameters will be determined:

- intermediate stagnation of waste and fibrous materials moving in the stream;

- $\quad$ hopper for collecting waste and fibrous materials Lc width of the lower channel;

- determine the distance $\mathrm{L}_{\mathrm{ox}}$ (between points $\mathrm{E}_{\mathrm{o}}$ and $\mathrm{E}$ ) from the point of inflow of the air flow to the point of the beginning of stagnation;

- Determination of the radii of curvature of the arc radii $R_{1}$ and $R_{2} \quad\left(R_{2}=R_{2}\left(E_{0} C_{o}\right)\right)$ $\left(R_{1}=R_{1}\left(C_{o} E\right)\right)$;

- determine the amount of waste and fibrous materials entering the collection bin;

- velocities $V_{1}$ and $V_{2}$ of upper and lower vertical currents after capture of waste.

To simplify the derivation of theoretical research results, we assume that the problem is two-dimensional and does not depend on time. When solving such problems, we rely on the methods of the theory of functions of a complex variable and ideal fluids $[4,5,6]$. The problem is solved in a parametric form. As an auxiliary field, we take the upper half-plane with a parametric variable and denote it as $\mathrm{G}_{\mathrm{z}}(\mathrm{t}=\xi+i \eta)$ (Fig. 5). In this case, according to the S.A.Chapligin singular point method, we reflect the complex potential field $\mathrm{G}_{\mathrm{W}}(\mathrm{W}=\varphi+i \Psi)$ (Fig. 4), the $\omega=\tau+i \theta ;\left(\tau=\ln \frac{V_{n o}}{V_{n}}\right)-$ N.E.Zhukovsky function, conformity to the $\mathrm{G}_{\mathrm{z}}(\mathrm{t}=\xi+i \eta)$ field (Fig. 5). In the reflection process, we assume that 
the boundary of the $G_{z}(z=x+i y)$ field is reflected (i.e., falls) on the true axis of the $G_{z}$ field $\operatorname{Re} G_{z}=\xi, \eta=0$. The relationship between $G_{z}$-field and $G_{w}$-field is adjustable as shown in Fig. 3 and 4.

In this case, by the method of Chapligin singular points $[4,5,6]$. The product of the $W(t)$ function by the $t=\xi+i \eta$ parameters will have first-order poles and zeros at points $A(t= \pm \infty), c(t=-1), E(t=e)$ and $D(t=1)$.

$\frac{d W}{d t}$ - we will plot this result by plotting a function at the poles and zeros:

$$
\frac{d W}{d t}=-\frac{q_{n}}{\pi} \frac{t-e}{t^{2}-1}, q_{n}=q_{c}+q_{d}=V_{n} L_{n 3} L_{n}=L_{A}
$$

in this: $q_{n}$ - (AA) flow rate of the mixture in the pipe (Fig. 2)

$q_{c}=q_{1}$ - air flow through the upper duct;

$q_{c}=q_{2}$ - the amount of fibrous waste along the bottom duct.

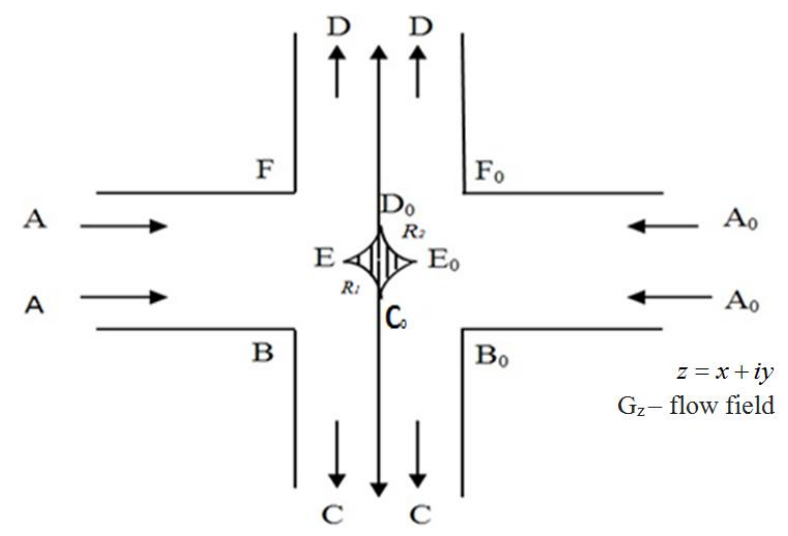

Fig. 2. Collision scheme of opposite currents $G_{z}$ - flow (field)

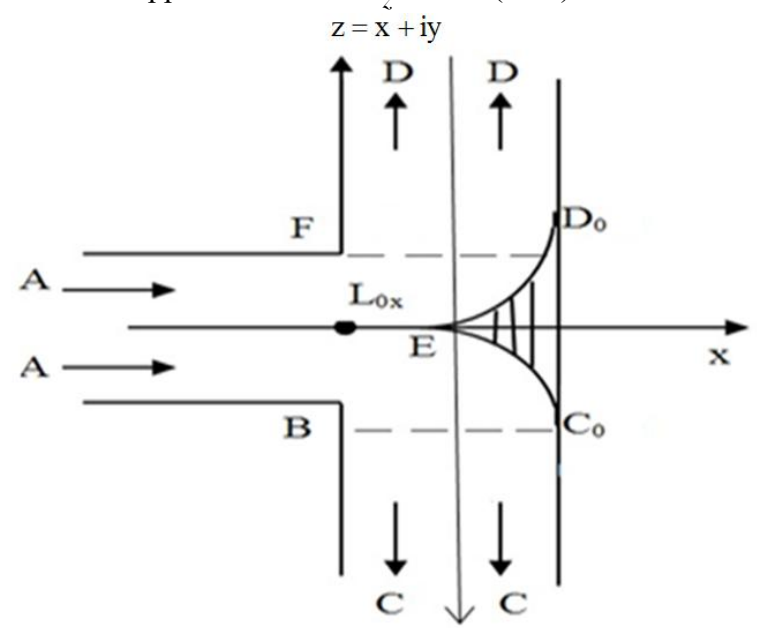

Fig.3. Scheme of the semi-sphere of the mixture flow $G_{z}$ - half of the stream 


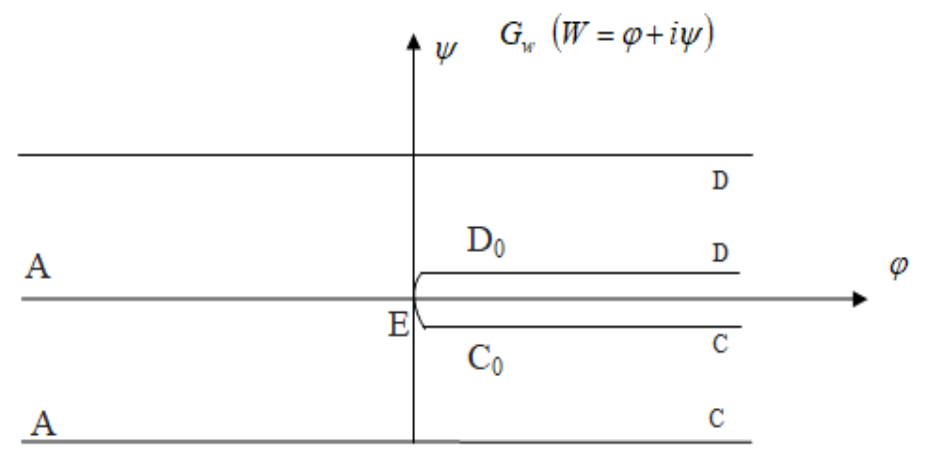

Fig.4. $G_{w}$ - complex potential field

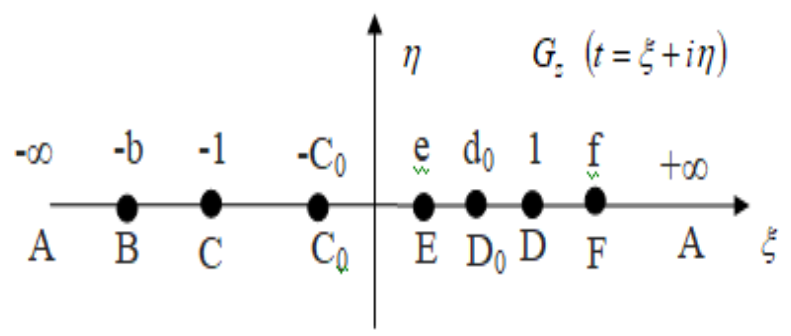

Fig.5. Canonical sphere

We determine the limit values of the N.E.Zhukovsky function:

in this $\frac{d W_{n}}{d z}=V_{n}, \quad \theta=\theta_{n}(t) . \quad(n=1 ; 2)$

$$
\omega_{n}(t)=\tau+i \theta, \tau=\ln \frac{V_{n 0}}{V_{n}}, \omega_{n}(t)=\ln \left|\frac{V_{n o}}{\frac{d W_{n}}{d z}}\right|
$$

We introduce a function similar to N.E.Zhukovsky's.

$$
\omega(z)=\ln \sqrt{\frac{\rho_{1} V_{10}{ }^{2}+\rho_{2} V_{20}{ }^{2}}{\rho_{1} V_{1}^{2}+\rho_{2} V_{2}^{2}}}
$$

in this $\theta=\theta(t)$ - the angle of deviation of the velocity vector;

$V_{n 0}$ - the velocity of the fibrous waste mixture with air;

$V_{1}$ - air speed;

$V_{2}$ - the velocity of the fibrous waste at the head of the pipe;

$V_{10}, V_{20}$ - respectively fiber emissions and air velocities along the ducts.

From (1) and (2) we obtain the following.

$$
\frac{V_{n o}}{V_{n}}=\sqrt{\frac{\rho_{1} V_{10}^{2}+\rho_{2} V_{20}^{2}}{\rho_{1} V_{1}^{2}+\rho_{2} V_{2}^{2}}} \quad \mathrm{n}=1.2
$$

In this case, the limit value of the N.E. Zhukovsky function has the following form. According to Fig. 5, $E=e, \mathrm{D}_{0}=d_{0}, \mathrm{~B}=-\mathrm{b}$ 


$$
\tau_{m} \omega_{n}(t)=\left\{\begin{array}{l}
0, \text { if },-\infty<\xi<-\mathrm{b}, \eta=0 \quad(A B), \\
\frac{-\pi}{2}, \text { if },-\mathrm{b}<\xi<-1, \eta=0 \quad(B C) \\
-\frac{\pi}{2}, \text { if },-1<\xi<C_{0}, \eta=0 \quad\left(C C_{O}\right) \\
\theta_{1}(\xi), \text { if }, \quad C_{O}<\xi<e, \quad \eta=0 \quad\left(C_{o} E\right) \\
\theta_{2}(\xi), \text { if }, \quad e<\xi<d_{o}, \quad \eta=0 \quad\left(E D_{0}\right) \\
\frac{\pi}{2}, \text { if }, d_{0}<\xi<1, \eta=0 \quad\left(D_{o} D\right) \\
\frac{\pi}{2}, \text { if }, 1<\xi<f, \eta=0(D F) \\
0, \text { if }, f<\xi<\infty, \quad \eta=0 \quad(F A)
\end{array}\right.
$$

Using the K. Schwartz integral formula, we obtain the following [3].

$$
\omega_{n}(\xi)=\frac{1}{\pi} \int_{-\infty}^{+\infty} \frac{\tau_{m} \omega_{n}(\xi) d \xi}{\xi-t}
$$

or, to put it more fully, from the boundary conditions of Zhukovsky's function:

$$
\begin{gathered}
\omega_{n}(\xi)=-\frac{1}{2} \int_{-b}^{-1} \frac{d \xi}{\xi-t}-\frac{1}{2} \int_{-1}^{-C_{o}} \frac{d \xi}{\xi-t}+\frac{1}{\pi} \int_{-C_{o}}^{e} \frac{\theta_{1}(\xi) d \xi}{\xi-t}+\frac{1}{\pi} \int_{e}^{d_{o}} \frac{\theta_{2}(\xi)}{\xi-t} d \xi+\frac{1}{2} \int_{d_{0}}^{1} \frac{d \xi}{\xi-t}+\frac{1}{2} \int_{1}^{f} \frac{d \xi}{\xi-t}= \\
=-\frac{1}{2} \ln \frac{-1-t}{-b-t}-\frac{1}{2} \ln \frac{-C_{o}-t}{-1-t}+\frac{1}{\pi} \int_{-C_{o}}^{e} \frac{\theta_{1}(\xi) d \xi}{\xi-t}+\frac{1}{\pi} \int_{e}^{d_{0}} \frac{\theta_{2}(\xi) d \xi}{\xi-t}+\frac{1}{2} \ln \frac{1-t}{d_{0}-t}+\frac{1}{2} \ln \frac{f-t}{1-t}= \\
=-\frac{1}{2} \ln \frac{1+t}{b+t} \cdot \frac{C_{0}+t}{1+t}+\frac{1}{2} \ln \frac{1-t}{d_{0}-t} \cdot \frac{f-t}{1-t}+I_{1}(t)+I_{2}(t)= \\
=-\frac{1}{2} \ln \frac{C_{0}+t}{b+t}+\frac{1}{2} \ln \frac{f-t}{d_{0}-t}+I_{1}(t)+I_{2}(t)= \\
=\frac{1}{2} \ln \frac{f-t}{d_{0}-t} \cdot \frac{b+t}{C_{0}+t}+I_{1}(t)+I_{2}(t)=\ln \sqrt{\frac{(t+b)(t-f)}{\left(t+C_{0}\right)\left(t-d_{0}\right)}}+I_{1}(t)+I_{2}(t)
\end{gathered}
$$

in this

$$
\begin{gathered}
I_{1}(t)=\frac{1}{\pi} \int_{C_{0}}^{e} \frac{\theta_{1}(\xi) d \xi}{\xi-t}, \quad I_{2}(t)=\frac{1}{\pi} \int_{e}^{d_{0}} \frac{\theta_{2}(\xi) d \xi}{\xi-t} \\
\theta_{2}(t)=A t+B \Rightarrow\left\{\begin{array}{l}
-\frac{\pi}{2}=-A C_{0}+B \\
0=A e+B
\end{array} \Rightarrow A\left(e+C_{0}\right)=\frac{\pi}{2} \Rightarrow A=\frac{\pi}{2\left(e+C_{0}\right)}\right. \\
B=-A e=-\frac{\pi e}{2\left(e+C_{0}\right)} \Rightarrow \theta_{2}(t)=\frac{\pi(t-e)}{2\left(e+C_{0}\right)}=\left\{\begin{array}{l}
0, \text { if }, t=e \\
-\frac{\pi}{2}, \text { if }, t=-C_{0}
\end{array}\right. \\
\theta_{1}(t)=A t+B ;\left\{\begin{array}{l}
0=A e+B \quad A=\frac{\pi}{2\left(d_{0}-e\right)}, \quad B=-\frac{\pi e}{2\left(d_{0}-e\right)} \\
\frac{\pi}{2}=A d_{0}+B \quad
\end{array}\right. \\
\theta_{1}(t)=\frac{\pi(t-e)}{2\left(d_{0}-e\right)}=\left\{\begin{array}{l}
0, \text { if }, t=e \\
\frac{\pi}{2}, i f, t=d_{0}
\end{array}\right.
\end{gathered}
$$


In this case, taking into account the formulas (5) - (7), we calculate $\mathrm{I}_{1}(\mathrm{t})$ and $\mathrm{I}_{2}(\mathrm{t}): \mathrm{t}=\xi+i \eta$, $\eta=0$

$$
\begin{aligned}
& I_{1}(t)=\frac{1}{\pi} \int_{-C_{0}}^{e} \frac{\pi(\xi-e) d \xi}{2\left(e+C_{0}\right) \cdot(\xi-t)}=\frac{1}{2\left(e+C_{0}\right)} \int_{-C_{0}}^{e} \frac{\xi-e}{\xi-t} d \xi=\frac{1}{2\left(e+C_{0}\right)} \int_{-C_{0}}^{e}\left(1+\frac{t-e}{\xi-t}\right) d \xi= \\
& =\frac{1}{2\left(e+C_{0}\right)}\left[\left(e+C_{0}\right)+(t-e) \ln \frac{e-t}{-C_{0}-t}\right]=\frac{1}{2}\left[1+\frac{t-e}{e+C_{0}} \ln \frac{t-e}{C_{0}+t}\right]=\frac{1}{2}+\ln \left(\frac{t-e}{C_{0}+t}\right)^{\frac{\tau-e}{2\left(e+C_{0}\right)}} \\
& I_{2}(t)=\frac{1}{\pi} \int_{e}^{d_{0}} \frac{\pi(\xi-e) d \xi}{2\left(d_{0}-e\right) \cdot(\xi-t)}=\frac{1}{2\left(d_{0}-e\right)} \int_{e}^{d_{0}} \frac{\xi-e}{\xi-t} d \xi=\frac{1}{2\left(d_{0}+e\right)} \int_{e}^{d_{0}}\left(1+\frac{t-e}{\xi-t}\right) d \xi= \\
& =\frac{1}{2\left(d_{0}+e\right)}\left[\left(d_{0}-e\right)+(t-e) \ln \frac{d_{0}-t}{e-t}\right]=\frac{1}{2}\left[1+\frac{t-e}{d_{0}-e} \ln \frac{d_{0}-t}{e-t}\right]=\frac{1}{2}+\ln \left(\frac{d_{0}-t}{e-t}\right)^{\frac{\tau-e}{2\left(d_{0}-e\right)}}
\end{aligned}
$$

In that case from (5):

$$
\begin{gathered}
\omega_{n}(t)=\ln \sqrt{\frac{(t+b)(t-f)}{\left(t+C_{0}\right)\left(t-d_{0}\right)}}+1+\ln \left(\frac{t-e}{C_{0}+t}\right)^{\frac{t-e}{2\left(e+C_{0}\right)}}+\ln \left(\frac{d_{0}-t}{e-t}\right)^{\frac{t-e}{2\left(d_{0}-e\right)}}= \\
=1+\ln \sqrt{\frac{(t+b)(t-f)}{\left(t+C_{0}\right)\left(t-d_{0}\right)}}+\ln \left(\frac{t-e}{C_{0}+t}\right)^{\frac{t-e}{2\left(e+C_{0}\right)}}+\ln \left(\frac{d_{0}-t}{e-t}\right)^{\frac{t-e}{2\left(d_{0}-e\right)}} \\
\omega_{n}(t)=1+\ln \sqrt{\frac{(t+b)(t-f)}{\left(t+C_{0}\right)\left(t-d_{0}\right)}} \cdot\left(\frac{t-e}{C_{0}+t}\right)^{\frac{t-e}{2\left(e+C_{0}\right)}} \cdot\left(\frac{d_{0}-t}{e-t}\right)^{\frac{t-e}{2\left(d_{0}-e\right)}}
\end{gathered}
$$

In this $1=\ln e_{1}, e_{1} \approx 2,71$

according to (2),

$$
V_{n}=V_{n 0} \sqrt{\frac{\left(t+C_{0}\right)\left(t-d_{0}\right)}{(t+b)(t-f)}} \cdot\left(\frac{C_{0}+t}{t-e}\right)^{\frac{t-e}{2\left(e+C_{0}\right)}} \cdot\left(\frac{e-t}{d_{0}-t}\right)^{\frac{t-e}{2\left(d_{0}-e\right)}} \cdot \frac{1}{2.71}
$$

we check the correctness of formula (9).

$$
\begin{gathered}
\text { 1. } y_{1}=\left(t+C_{0}\right)^{\frac{1}{2}} \cdot\left(C_{0}+t\right) \frac{t-e}{2\left(e+C_{0}\right)}=\left(t+C_{0}\right) \frac{t-e}{2\left(e+C_{0}\right)} \\
\ln y_{1}=\frac{1}{2\left(e+C_{0}\right)}\left(t+C_{0}\right) \ln \left(t+C_{0}\right) \Rightarrow \ln y_{1}=\frac{1}{2\left(e_{1}+C_{0}\right)} \lim _{t \rightarrow-C_{0}}\left(t+C_{0}\right) \ln \left(t+C_{0}\right)= \\
=\frac{1}{2\left(e+C_{0}\right)} \lim _{t \rightarrow-C_{0}} \frac{\ln \left(t+C_{0}\right)}{\frac{1}{t+C_{0}}}=\left(\frac{\infty}{\infty}\right)
\end{gathered}
$$

according to the Lopital rule,

$$
\frac{1}{2\left(e+C_{0}\right)} \lim _{t \rightarrow-C_{0}} \frac{\frac{1}{t+C_{0}}}{-\frac{1}{\left(t+C_{0}\right)^{2}}}=-\frac{1}{2\left(e+C_{0}\right)} \lim _{t \rightarrow-C_{0}}\left(t+C_{0}\right)=0
$$

$\ln y_{1}=0, y_{1}=e^{0}=1$

$$
\begin{aligned}
& \text { 2. } y_{2}=\left(t-d_{0}\right)^{\frac{1}{2}} \cdot\left(d_{0}-t\right)^{\frac{t-e}{2\left(d_{0}-e\right)}}=\left(d_{0}-t\right)^{\frac{d_{0}-t}{2\left(d_{0}-e\right)}} \\
& \ln y_{2}=\frac{1}{2\left(d_{0}-e\right)}\left(d_{0}-t\right) \ln \left(d_{0}-t\right)=\frac{1}{2\left(d_{0}-e\right)} \cdot \frac{\ln \left(d_{0}-t\right)}{\frac{1}{d_{0}-t}}
\end{aligned}
$$




$$
\begin{gathered}
\lim _{t \rightarrow-d_{0}} \ln y_{2}=\frac{1}{2\left(d_{0}-e\right)} \lim _{t \rightarrow d_{0}} \frac{-\frac{1}{d_{0}-t}}{-\frac{1}{\left(d_{0}-t\right)^{2}}}=\frac{1}{2\left(d_{0}-e\right)} \lim _{t \rightarrow d_{0}}\left(d_{0}-t\right)=0 \\
\ln y_{2}=0, y_{2}=e^{0}=1 ; y_{1}=y_{2}
\end{gathered}
$$

Using formulas (1), (2) and (9) to determine the geometric expressions of the problem, we obtain:

$$
\frac{d z}{d t}=\frac{d z}{d W} \cdot \frac{d W}{d t}=-\frac{q_{n}}{\pi} \frac{t-e}{t^{2}-1} \cdot \frac{1}{\frac{d W}{d t}}
$$

henceforth;

$$
\frac{d z}{d t}=-\frac{V_{n} L_{n}}{\pi} \cdot \frac{t-e}{t^{2}-1} \cdot \frac{1}{V_{n_{0}}} \cdot \sqrt{\frac{(t+b)(t-f)}{\left(t+C_{0}\right)\left(t-d_{0}\right)}} \cdot \sqrt{2,71} \cdot\left(\frac{d_{0}-t}{e-t}\right)^{\frac{t-e}{2\left(d_{0}-e\right)}}
$$

Also,

$$
\frac{d z}{d t}=-\sqrt{2.71} \frac{L_{n} F}{\pi} \cdot \frac{t-e}{t^{2}-1} I_{10}(t) \cdot I_{20}(t)
$$

in this $I_{10}(t)=\sqrt{\frac{(t+b)(t-f)}{\left(t+C_{0}\right)\left(t-d_{0}\right)}}, L_{n}$ - (AA) the starting width of the pipe

$$
\begin{gathered}
I_{20}(t)=\left(\frac{t-e}{C_{0}+t}\right)^{\frac{t-e}{2\left(e+C_{0}\right)}} \cdot\left(\frac{d_{0}-t}{e-t}\right)^{\frac{t-e}{2\left(d_{0}-e\right)}} \\
F=\sqrt{\frac{\rho_{1} V_{10}^{2}+\rho_{2} V_{20}^{2}}{\rho_{1} V_{1}^{2}+\rho_{2} V_{2}^{2}}}=\frac{V_{10}}{V_{1}} \sqrt{\frac{1+\hat{\rho}_{2} g_{1}}{1+\hat{\rho}_{2} g_{2}}}
\end{gathered}
$$

If we take into account the concentration of $f_{1}+f_{2}=1$ phases, then we obtain the following. According to (3),

$$
F=\hat{V}_{1} \sqrt{\frac{\left(1-f_{2}\right)^{2}+f_{2}^{2} \hat{\rho}_{2} g_{1}}{1+\hat{\rho}_{2} g_{2}}}
$$

in this: $\hat{\rho}_{2}=\frac{\rho_{2}}{\rho_{1}} ; \rho_{1}$ and $\rho_{2}$ - densities of fibrous waste and air at the head of the pipe $\hat{V}_{1}=\frac{V_{10}}{V_{1}} V_{10}$ and $V_{1}(\mathrm{AA})$ air velocities at the top of the pipe and along the vertical channel

$\hat{g}_{1}=\left(\frac{V_{20}}{V_{10}}\right)^{2}, \hat{g}_{2}=\left(\frac{V_{2}}{V_{1}}\right)^{2} V_{20}$ and $V_{2}$ - respectively (AA) the velocities of the fibrous mixtures at the beginning of the channel and along the bottom channel.

Consequently, the law of interaction between air and fibrous mixtures from two opposite pipes: (AA) is directly related to the density of fibrous air in the pipe head and the velocities of air and fibrous mixtures in the channels, which is proved in the above calculations. 


\section{Results and Discussion}

It can be seen that expressions (1), (9), (10) and (11) (AA) allow one to accurately express the law of interaction between air and a fibrous mixture leaving the pipe. Now let's calculate the parameters of movement in the flow of air-fiber mixtures in two opposite pipes.

Situation 1. In this case, we perform the following calculations: $\mathrm{g}_{1}=0.33$; Let be $\mathrm{g}_{2}=1$; $\mathrm{L}_{\mathrm{A}}=0.4$,

Table 1. $\mathrm{g}_{1}=0.33 ; \mathrm{g}_{2}=1 ; \mathrm{L}_{\mathrm{A}}=0.4$ results of calculation of values

\begin{tabular}{|c|l|c|l|l|c|c|l|}
\hline № & $\overline{\boldsymbol{\rho}}_{\mathbf{2}}$ & \multicolumn{1}{|c}{$\mathbf{F}$} & \multicolumn{1}{|c|}{$\mathbf{R}_{\mathbf{1}}$} & $\overline{\boldsymbol{R}}_{\mathbf{1}}$ & $\overline{\boldsymbol{L}}_{\boldsymbol{c}}$ & $\mathbf{L}_{\mathbf{c}}$ & $\mathbf{L}_{\mathbf{o x}}=\mathbf{L}_{\mathbf{c}}-\mathbf{R}_{\mathbf{1}}$ \\
\hline 1 & 0.08 & 0.974869 & 4.510788 & 0.11277 & 0.558794 & 22.35176 & 17.84097 \\
\hline 2 & 0.1 & 0.969067 & 4.48394 & 0.1121 & 0.555468 & 22.21872 & 17.73478 \\
\hline 3 & 0.12 & 0.963439 & 4.457898 & 0.11145 & 0.552242 & 22.08968 & 17.63178 \\
\hline 4 & 0.14 & 0.957977 & 4.432624 & 0.11082 & 0.549111 & 22.96444 & 17.53182 \\
\hline
\end{tabular}

Here, $\bar{R}_{1}$ - radius of curvature, $\bar{L}_{c}$ - channel width, $\mathrm{L}_{\mathrm{ox}}-$ the distance between the inner horizontal pipe and the $\mathrm{E}$ point of division of the flow.

Situation 2. Let be $\mathrm{g}_{1}=0.5 ; \mathrm{g}_{2}=1 ; \mathrm{L}_{\mathrm{A}}=0.4$,

Table 2. $\mathrm{g}_{1}=0.5 ; \mathrm{g}_{2}=1 ; \mathrm{L}_{\mathrm{A}}=0.4$ results of calculation of values

\begin{tabular}{|l|l|l|l|l|l|l|l|}
\hline № & $\overline{\boldsymbol{\rho}}_{\mathbf{2}}$ & \multicolumn{1}{|c|}{$\mathbf{F}$} & $\mathbf{R}_{\mathbf{1}}$ & $\overline{\boldsymbol{R}}_{\mathbf{1}}$ & \multicolumn{1}{c|}{$\overline{\boldsymbol{L}}_{\boldsymbol{c}}$} & \multicolumn{1}{c|}{$\mathbf{L}_{\mathbf{c}}$} & $\mathbf{L}_{\mathbf{o x}}=\mathbf{L}_{\mathbf{c}}-\mathbf{R}_{\mathbf{1}}$ \\
\hline 1 & 0.08 & 0.981307 & 4.540574 & 0.11351 & 0.562484 & 22.49936 & 17.95878 \\
\hline 2 & 0.1 & 0.977008 & 4.520685 & 0.11302 & 0.56002 & 22.4008 & 17.88012 \\
\hline 3 & 0.12 & 0.972846 & 4.501424 & 0.11254 & 0.557634 & 22.30536 & 17.80394 \\
\hline 4 & 0.14 & 0.968812 & 4.482759 & 0.11207 & 0.555322 & 22.21287 & 17.73012 \\
\hline
\end{tabular}

Situation 3. Let be $\mathrm{g}_{1}=0.95 ; \mathrm{g}_{2}=1 ; \mathrm{L}_{\mathrm{A}}=0.4$,

Table 3. $\mathrm{g}_{1}=0.95 ; \mathrm{g}_{2}=1 ; \mathrm{L}_{\mathrm{A}}=0.4$ results of calculation of values

\begin{tabular}{|l|l|c|c|c|c|c|l|}
\hline № & $\overline{\boldsymbol{\rho}}_{\mathbf{2}}$ & $\mathbf{F}$ & $\mathbf{R}_{\mathbf{1}}$ & $\overline{\boldsymbol{R}}_{\mathbf{1}}$ & $\overline{\boldsymbol{L}}_{\boldsymbol{c}}$ & $\mathbf{L}_{\mathbf{c}}$ & $\mathbf{L}_{\mathbf{o x}}=\mathbf{L}_{\mathbf{c}}-\mathbf{R}_{\mathbf{1}}$ \\
\hline 1 & 0.08 & 0.998146 & 4.618492 & 0.11546 & 0.572136 & 22.88546 & 18.26696 \\
\hline 2 & 0.1 & 0.997725 & 4.616541 & 0.11541 & 0.571895 & 22.87579 & 18.25924 \\
\hline 3 & 0.12 & 0.997318 & 4.614658 & 0.11537 & 0.571661 & 22.86646 & 18.2518 \\
\hline 4 & 0.14 & 0.996925 & 4.612841 & 0.11532 & 0.571436 & 22.85745 & 18.24461 \\
\hline
\end{tabular}

Now, we calculate the arithmetic mean of the results obtained from Table 1:

$\mathrm{R}_{1}($ average $)=\frac{4.510788+4.48394+4.457898+4.432624}{4} \approx 4.4713 \mathrm{sm}$

$\mathrm{L}_{\mathrm{c}}($ average $)=\frac{22.35176+22.21872+22.08968+21.9644}{4} \approx 22.156 \mathrm{sm}$

$\mathrm{L}_{\text {ox }}($ average $)=22.156-4.4713 \approx 17.68 \mathrm{sm}$

We calculate the arithmetic mean of the results obtained from Table 2:

$\mathrm{R}_{1}($ average $)=\frac{4.540574+4.520685+4.501424+4.482759}{4} \approx 4.5113 \mathrm{sm}$

$\mathrm{L}_{\mathrm{c}}($ average $)=\frac{22.49936+22.4008+22.30536+22.21287}{4} \approx 22.3545 \mathrm{sm}$ 
$\mathrm{L}_{\text {ox }}($ average $)=22.3545-4.5113 \approx 17.84 \mathrm{sm}$

We calculate the arithmetic mean of the results obtained from Table 3:

$$
\begin{aligned}
& \mathrm{R}_{1}(\text { average })=\frac{4.618492+4.616541+4.614658+4.612841}{4} \approx 4.6156 \mathrm{sm} \\
& \mathrm{L}_{\mathrm{c}}(\text { average })=\frac{22.88546+22.87579+22.86646+22.85745}{4} \approx 22.8712 \mathrm{sm} \\
& \mathrm{L}_{\text {ox }}(\text { average })=22.8712-4.6156 \approx 18.25 \mathrm{sm}
\end{aligned}
$$

Now, we calculate the average values of the results obtained from Tables 1, 2, 3 above:

$$
\begin{gathered}
\left.R_{1} \text { (average }\right)=\frac{R_{1}(\text { average } 1-\text { table })+R_{1}(\text { average } 2-\text { table })+R_{1}(\text { average } 3-\text { table })}{3}= \\
=\frac{4.4713+4.5113+4.6156}{3} \approx 4.53 \mathrm{sm} \\
R_{1}(\text { average })=\frac{R_{1}(\text { average } 1-\text { table })+R_{1}(\text { average } 2-\text { table })+R_{1}(\text { average } 3-\text { table })}{3}= \\
=\frac{22.156+22.354+22.871}{3} \approx 22.46 \mathrm{sm} \\
L_{C}(\text { average })=\frac{R_{C}(\text { average } 1-\text { table })+R_{C}(\text { average } 2-\text { table })+R_{C}(\text { average } 3-\text { table })}{3}= \\
=\frac{17.68+17.84+18.25}{3} \approx 17.93 \mathrm{sm} \\
\mathrm{L}_{\text {ox }}(\text { average })=\frac{\left(\mathrm{L}_{\text {ox }}(\text { average } 1-\text { table })+\mathrm{L}_{\text {ox }}(\text { average } 2-\text { table })+\mathrm{L}_{\text {ox }}(\text { average } 3-\text { table })\right.}{3}
\end{gathered}
$$

\section{Conclusions}

Based on a comparison of the cases, we obtain the radius of curvature of the $\operatorname{arcs} \mathrm{S}_{0} \mathrm{E}=\mathrm{ED}_{0}$ $R_{1} \approx 4.53 \mathrm{sm}$, as well as the width of the $L_{C} \approx 22.46 \mathrm{sm}$ channel and the distance $L_{o x} \approx 17.93 \mathrm{sm}$ from the pipe to the point when two media are separated.

The above, mathematically and theoretically substantiated existing parameters of the flow of air and mixtures of fibers in opposite pipes, can be taken as a scientific and theoretical basis for solving the problem of flowing around air and separating from it mixtures of fibers isolated from technological processes cotton ginning.

\section{References}

1. K. E. Kenyon, Fluid Reaction Force on Solids, Natural Science 10, 416-419 (2018)

2. W. Peukert, C. Wadenpohl, Industrial separation of fine particles with difficult dust properties, Powder Technology 118, 136-148 (2001)

3. M. Khodjiev, I. Abbazov, O. Alimov, J. Karimov, Fraction Structure of Cotton Cleaning Equipment in Cotton Enterprises and their Cleaning Effectiveness, Int. J. Advanced Research in Science, Engineering and Technology 6, 7983-7988 (2019) 
4. V. S. Kuznetsov, S. V. Denisov, Pneumatic transport at wood processing enterprises, External Pneumatic Conveying Units, Bratsk (2007)

5. K. Hoganson, D. Gross, Patent USA, Variable speed drive for pneumatic transport system, US 7,950,879 B2. 05/31/2011.

6. M. T. Xodjiev, Development of the theory and substantiation of the parameters of the mechanized technological process of minimum-modular compaction and storage of raw cotton, Doctor of Technical Science Dissertation, Tashkent (1998)

7. V. S. Gurev, V. A. Uspenskii, Optimum gas velocity when entering the cyclone, Industrial and Sanitary Gas Cleaning 4, 12-14 (1975)

8. I. Z. Abbazov, Impact device for collecting fiber waste on dust trapping, Textile Journal of Uzbekistan 7(1), 28-35 (2019)

9. M. T. Khojiev, I. Z. Abbasov, B. M. Mardonov, Theoretical study of the motion of dust particles in the chamber of the collector, Journal of Textile Problems 75-79 (2015)

10. N. Yu. Tashlanov, V. U. Usmanov, M. R. Raximov, On the efficiency of using a wet dust collector in cotton ginning plantsx, Cotton Industry 4, 16-19 (1973)

11. M. T. Xodjiyev, O. N. Alimov, I. Z. Abbazov, Potential structure of dust coming out of process of cotton reproduction, Scientific-Technical Journal 23, 34-41 (2019)

12. F. O. Egamberdiev, K. J. Jumaniyazov, I. Z. Abbazov, Study of the influence of the guiding device on increasing the efficiency of fiber cleaning, IOP Conference Series: Earth and Environmental Science 614, 012123 (2020)

13. H. Yu. Ulugmuradov, I. Z. Abbazov, R. M. Muradov, Study on improving the efficiency of cleaning the pile drum, IOP Conference Series: Earth and Environmental Science 614, 012127 (2020) 